\title{
A modified implicit-explicit integration scheme: an application to elastoplasticity problems
}

\author{
Plínio G. C. Prazeres ${ }^{1}$ Luís A. G. Bitencourt Jr. ${ }^{1}$ Túlio N. Bittencourt ${ }^{1}$ • \\ Osvaldo L. Manzoli ${ }^{2}$
}

Received: 11 February 2015 / Accepted: 17 March 2015 / Published online: 29 March 2015

(C) The Brazilian Society of Mechanical Sciences and Engineering 2015

\begin{abstract}
In this paper, a novel modified version of the implicit-explicit integration scheme (IMPL-EX) proposed by Oliver (Comput Methods Appl Mech Eng 197:1865-1889, 2008) is presented. This modified approach has as its main difference with respect to the standard IMPL-EX the choice of internal variables to be updated. In the modified approach what is updated are the plastic strain tensor components, instead of the plastic multiplier. This choice of update, as will be shown, has the advantage of making the algorithm stiffness matrix constant, while keeping all the advantages that comes with the standard IMPL-EX already pointed out by Oliver in (Comput Methods Appl Mech Eng 197:1865-1889, 2008) and (Comput Methods Appl Mech Eng 195:7093$7114,2006)$. The scope of the paper will be restricted to the application of the proposed scheme to elastoplasticity problems. Due to what is exposed above the authors named the proposed method by Modified IMPL-EX. To assess the algorithm proposed, a few verification examples, with closedform solutions, for elastoplasticity problems are presented and the Modified IMPL-EX results are compared with the results obtained by the implicit integration scheme.
\end{abstract}

Keywords Implicit-explicit integration scheme · Non-linear elastoplasticity problem $\cdot$ Finite element method

Technical Editor: Fernando Alves Rochinha.

Plínio G. C. Prazeres

plinioglauber@usp.br

1 University of São Paulo, Av. Prof. Luciano Gualberto, Trav. 3 n. 380, São Paulo, SP CEP-05508-010, Brazil

2 São Paulo State University, Av. Eng. Luiz Edmundo C. Coube 14-01, Bauru, SP CEP-17033-360, Brazil

\section{Introduction}

Based in the implicit-explicit integration scheme (IM-EX) proposed by Oliver ([5]), in this paper, a modified version of the IMPL-EX scheme is presented.

Likewise the standard IMPL-EX scheme (see also [4]), the proposed scheme consists of using two integration scheme in the same time step: an standard implicit (Backward-Euler) scheme furnishing "implicit" values of stresses $\boldsymbol{\sigma}_{n+1}\left(\boldsymbol{\varepsilon}_{n+1}\right)$ in terms of the current strains (more details in Sect. 4) and an explicit integration scheme furnishing other "explicit" values of the stresses $\tilde{\boldsymbol{\sigma}}_{n+1}\left(\boldsymbol{\varepsilon}_{n+1}\right)$ (also to be detailed in Sect. 4 below).

In [4] it is argued that, since the extrapolated values of the plastic multiplier $\Delta \tilde{\lambda}_{n+1}$ are obtained in terms of the implicit integrated values, $\Delta \lambda_{n}$ and $\Delta \lambda_{n-1}$, one can expect that the well-known stability properties of the implicit integration procedures are being inherited by their explicit extrapolations. Likewise, the same argument can be made in favor of the proposed scheme.

Another property pointed out in [4], that is automatically inherited by the Modified IMPL-EX, is the robustness (stability) of the standard IMPL-EX integration scheme. To explain that, in [4] it is said that, the IMPL-EX "can be understood as composed of a prediction stage, at the end of time step $n$, followed by a linear correction, characterized by the constant and positive definite operator" $\tilde{\mathbf{C}}^{\text {tan }}\left(\tilde{\boldsymbol{\sigma}}_{n+1}\right)$, "during time step $n+1$ ". Such property derives in an algorithmic stiffness matrix always positive definite resulting that "no numerical instabilities should be expected at any time and the robustness of the analysis should be dramatically increased". Also, it is said in [4] that "since the structural tangent stiffness matrix is now constant and well conditioned, the Newton-Raphson procedure should converge in just one iteration per time step". 
Of course the drawbacks existents in the standard IMPL-EX are also inherited by the Modified IMPLEX scheme and the main one is that the method delivers "smaller accuracies for relatively large time steps, in comparison with the ones obtained with the purely implicit integration procedure", as pointed out in [4].

To show that the robustness of the standard IMPLEX scheme, alied to the use of an specific symmetric version of the elementary enriched finite element in the context of a problem of Continuum Strong Discontinuity Approach, in [4] a benchmark problem of a double cantilever beam test where the lack of stability and robustness of the problem is used to illustrate the benefits of the standard IMPL-EX is presented. Then, to show the accuracy and the computational advantage of the method, in [4], an elastoplastic problem of slope instability is evaluated.

With the same purpose, the assessment of the robustness, accuracy and computational cost, in [5], two examples, one consisting of the plastic loading of a rectangular plate, with a center hole, vertically stretched and another where a rectangular plate discretized in five rows of quads and pushed horizontally along the upper rows, inducing a pure shear stress state in the lowest row of elements, are analyzed.

With that in mind, in this paper, in Sect. 5, a series of representative examples are used to assess numerically the properties of the Modified IMPL-EX integration scheme, in terms of its accuracy, computational cost and robustness.

\section{Displacement driven algorithm}

The problem posed in this study is the one of a displacement-driven, material non-linear finite element equation in which incremental-iterative methods based on variants of the Newton-Raphson method are used to obtain a solution, according to the following balance equation:

$$
\begin{aligned}
\mathbf{R}_{n+1}\left(\mathbf{u}_{n+1}, \boldsymbol{\sigma}_{n+1}\left(\mathbf{u}_{n+1}\right)\right)= & \mathbf{F}_{\text {int }}\left(\boldsymbol{\sigma}_{n+1}\left(\mathbf{u}_{n+1}\right)\right)+ \\
& -\mathbf{F}_{\text {ext }}\left(t_{n+1}\right)=\mathbf{0}
\end{aligned}
$$

where $\mathbf{F}_{\text {int }}$ are the internal forces, $\mathbf{F}_{\text {ext }}$ are the external forces and $\boldsymbol{\sigma}_{n+1}$ and $\mathbf{u}_{n+1}$ are, respectively, the stresses and the nodal displacements at a representative time step $t_{n+1}$ that can be identified as the loading factor or the arc length parameter playing the role of time in quasi-static problems.

The incremental equation for the residuum $\mathbf{R}_{n+1}$ may be expressed as follows:

$\mathbf{R}_{n+1}=\mathbf{R}_{n}+\mathbf{K}_{n+1} \Delta \mathbf{u}_{n+1}=\mathbf{0}$

where $\Delta \mathbf{u}_{n+1}$ are the displacement increments, and the tangent stiffness matrix $\mathbf{K}_{n+1}$ is computed as follows:

$$
\begin{aligned}
\mathbf{K}_{n+1} & =\frac{\partial \mathbf{F}_{\text {int }}\left(\mathbf{u}_{n+1}\right)}{\partial \mathbf{u}_{n+1}} \\
& =\mathbf{A}_{e=1, \ldots, n^{e l}}\left(\int_{\Omega^{e l}} \mathbf{B}^{e l^{T}} \mathbf{C}_{n+1}^{\tan }\left(\boldsymbol{\sigma}_{n+1}\left(\mathbf{u}_{n+1}\right)\right) \mathbf{B}^{e l} d \Omega\right)
\end{aligned}
$$

where $\mathbf{A}$ is the assembling operator, $\mathbf{C}_{n+1}^{\tan }\left(\boldsymbol{\sigma}_{n+1}\right)=\frac{\partial \boldsymbol{\sigma}_{n+1}}{\partial \boldsymbol{\varepsilon}_{n+1}}$ is the material tangent constitutive matrix and $\mathbf{B}^{e l}=\nabla \mathbf{N}^{e l}$ is the standard strain-displacement matrix, evaluated at the element (el) level, where $\mathbf{N}^{e l}$ is the matrix of shape functions of the element and $\nabla$ is a differential operator.

\section{Classical elastoplasticity}

According to elastoplasticity theory (see, e.g., [1, 6-9]), if the load exceeds a certain range, not only does elastic strain occur but plastic (non-recoverable) strain does as well such that

$\boldsymbol{\varepsilon}=\boldsymbol{\varepsilon}^{e}+\boldsymbol{\varepsilon}^{p}$

in total quantities or

$\Delta \boldsymbol{\varepsilon}=\Delta \boldsymbol{\varepsilon}^{e}+\Delta \boldsymbol{\varepsilon}^{p}$

in incremental quantities, which are more suitable for finite element codes. In Eqs. 4 and 5, the superscripts $e$ and $p$ denote elastic and plastic behavior, respectively.

Physically, the increments in stress $\Delta \sigma$ is associated with the increments in elastic strain as follows:

$\Delta \boldsymbol{\sigma}=\mathbf{C} \Delta \boldsymbol{\varepsilon}^{e}=\mathbf{C}\left(\Delta \boldsymbol{\varepsilon}-\Delta \boldsymbol{\varepsilon}^{p}\right)$

where $\mathbf{C}$ is the material elastic constitutive matrix.

The range beyond which plastic deformation takes place is generally designated by a yield criterion $F$ such that

$F=F(\sigma, \alpha)=0$

where $\boldsymbol{\alpha}$ is a hardening parameter that depends on the history of deformation.

How the plastic strain increments $\Delta \boldsymbol{\varepsilon}^{p}$ evolve during plastic flow is determined by the so-called flow rule,

$\Delta \boldsymbol{\varepsilon}^{p}=\Delta \lambda \frac{\partial Q}{\partial \boldsymbol{\sigma}}$

expressed in terms of a potential function $Q=Q(\boldsymbol{\sigma}, \boldsymbol{\alpha})=0$, which has units of stress, called the plastic potential and the parameter $\Delta \lambda$ which is a scalar usually called the "plastic multiplier". The flow rule is considered to be "associated" if $Q=F$ and "non-associated" otherwise. Note that, in Eq. 8, the direction of the plastic strain increments $\Delta \boldsymbol{\varepsilon}^{p}$ is given by the plastic potential $\frac{\partial Q}{\partial \sigma}$, while its magnitude is given by the plastic multiplier $\Delta \lambda$.

Meanwhile, by definition, $\Delta \lambda \geq 0$. Physically, the admissible range of stresses is limited to $F \leq 0$ and plastic flow can only take place on the yield surface (i.e., for 
$F=0$ ). As a consequence (according to Eq. 8), if $\Delta \lambda=0$ , then $F<0$ and if $F=0$, then $\Delta \lambda>0$. Therefore, one can write the following Kuhn-Tucker conditions,

$\Delta \lambda \geq 0 ; \quad F \leq 0$

$$
\Delta \lambda F=0
$$

and because $F=0$ during plastic flow, one can express the consistency (or persistency) condition as follows:

$\Delta \lambda d F=0 \quad($ if $F=0)$

from which the plastic multiplier $\Delta \lambda$ can be determined.

\subsection{Implicit integration scheme}

In view of Eqs. 4-10, the algorithm for implicitly integrating elastoplastic models over the time interval $\left[t_{n}, t_{n+1}\right]$ and for one integration point at a time is summarized in the following steps:

1. Initially, the trial stresses are computed as follows:

$$
\boldsymbol{\sigma}_{n+1}^{\text {trial }}=\boldsymbol{\sigma}_{n}+\mathbf{C} \Delta \boldsymbol{\varepsilon}_{n+1}
$$

2. Then, the yield function values, Eq. 7, are evaluated for the trial stresses $\boldsymbol{\sigma}_{n+1}^{\text {trial }}$ as follows:

$F=F^{\text {trial }}\left(\boldsymbol{\sigma}_{n+1}^{\text {trial }}, \boldsymbol{\alpha}_{n}\right)$

The inequality $F^{\text {trial }}<0$ indicates that an elastic/ unloading state has taken place, and therefore,

$$
\begin{aligned}
& \Delta \lambda_{n+1}=0 \\
& \Delta \boldsymbol{\varepsilon}_{n+1}^{p}=\mathbf{0} \\
& \mathbf{C}_{n+1}^{\mathrm{tan}}=\mathbf{C} \\
& \boldsymbol{\sigma}_{n+1}=\boldsymbol{\sigma}_{n+1}^{\text {trial }}
\end{aligned}
$$

and one should return to step 1 for the next integration point. However, if $F^{\text {trial }} \geq 0$, then plastic strain increments $\Delta \varepsilon_{n+1}^{p}$ are being developed $(\Delta \lambda>0)$ and one should compute $\Delta \lambda_{n+1}$ using Eqs. 9 and 10 according to steps iii to iv.

3. In a plastic state $\left(F\left(\boldsymbol{\sigma}_{n+1}^{\text {trial }}, \boldsymbol{\alpha}_{n}\right) \geq 0\right)$, the increment in plastic strains $\Delta \boldsymbol{\varepsilon}^{p}$ should be computed according to Eq. 8. Then, the problem becomes one of solving an implicit system of non-linear equations, shown below, yielding $\boldsymbol{\sigma}_{n+1}, \Delta \lambda_{n+1}, \boldsymbol{\alpha}_{n+1}, \Delta \boldsymbol{\varepsilon}_{n+1}^{p}$.

$$
\begin{aligned}
F\left(\boldsymbol{\sigma}_{n+1}, \boldsymbol{\alpha}_{n+1}\right) & =0 \\
\Delta \boldsymbol{\varepsilon}_{n+1}^{p} & =\Delta \lambda_{n+1} \frac{\partial Q\left(\boldsymbol{\sigma}_{n+1}\right)}{\partial \boldsymbol{\sigma}_{n+1}} \\
\boldsymbol{\sigma}_{n+1} & =\boldsymbol{\sigma}_{n+1}^{\text {trial }}-\mathbf{C} \Delta \boldsymbol{\varepsilon}_{n+1}^{p} \\
\boldsymbol{\alpha}_{n+1}\left(\Delta \lambda_{n+1}\right) & =\boldsymbol{\alpha}_{n}+\boldsymbol{\varphi}_{n+1}\left(\Delta \lambda_{n+1}\right)
\end{aligned}
$$

where $\boldsymbol{\alpha}(\Delta \lambda)$ represents the hardening parameter (according to the hardening law) and $\varphi_{n+1}\left(\Delta \lambda_{n+1}\right)$ is a vector (for general cases) with dimensions equal to those of $\boldsymbol{\alpha}_{n+1}$, a function of the plastic multiplier $\Delta \lambda_{n+1}$. The most common approach to solve an implicit system of non-linear equations, like the Eq. 14 above, is the Newton-Raphson method, used here at the integration point level.

4. Then, the internal variables (the total plastic strains $\boldsymbol{\varepsilon}_{n+1}^{p}$ and the total stresses $\left.\sigma_{n+1}\right)$ are updated as follows:

$$
\begin{aligned}
& \boldsymbol{\varepsilon}_{n+1}^{p}=\boldsymbol{\varepsilon}_{n}^{p}+\Delta \boldsymbol{\varepsilon}_{n+1}^{p} \\
& \boldsymbol{\sigma}_{n+1}=\boldsymbol{\sigma}_{n+1}^{\text {trial }}-\mathbf{C} \Delta \boldsymbol{\varepsilon}_{n+1}^{p}=\mathbf{C}\left(\boldsymbol{\varepsilon}_{n+1}-\boldsymbol{\varepsilon}_{n+1}^{p}\right)
\end{aligned}
$$

and one should proceed to step 5.

5. Finally, the algorithmic tangent operator $\mathbf{C}_{n+1}^{\mathrm{tan}}\left(\boldsymbol{\sigma}_{n+1}\right)$ should be computed, as follows:

$\mathbf{C}_{n+1}^{\mathrm{tan}}\left(\boldsymbol{\sigma}_{n+1}\right)=\frac{\partial \boldsymbol{\sigma}_{n+1}}{\partial \boldsymbol{\varepsilon}_{n+1}}$

and one should return to step 1 for the next integration point.

\section{IMPL-EX and Modified IMPL-EX Integration Schemes Updates}

In the formulation of the IMPL-EX integration scheme ([5]), previously mentioned, the update of the internal variables, in elastoplasticity problems, is accomplished by the explicit evaluation of the plastic multiplier $\Delta \lambda$ introduced in Eq. 8.

In other words, the update of the internal variables (the plastic multiplier $\Delta \lambda_{n+1}$, the total plastic strains $\varepsilon_{n+1}^{p}$ and the total stresses $\sigma_{n+1}$ ), here, the IMPL-EX plastic multiplier $\Delta \tilde{\lambda}_{n+1}$, the IMPL-EX total plastic strains $\tilde{\boldsymbol{\varepsilon}}_{n+1}^{p}$ and the IMPL-EX total stresses $\tilde{\boldsymbol{\sigma}}_{n+1}$, in Eq. 17 below, are obtained as follows,

$$
\begin{aligned}
\tilde{\boldsymbol{\alpha}}_{n+1}\left(\Delta \tilde{\lambda}_{n+1}\right) & =\boldsymbol{\alpha}_{n}+\boldsymbol{\varphi}_{n}\left(\Delta \tilde{\lambda}_{n+1}\right) \\
\tilde{\boldsymbol{\varepsilon}}_{n+1}^{p} & =\boldsymbol{\varepsilon}_{n}^{p}+\Delta \tilde{\lambda}_{n+1} \mathbf{C} \frac{\partial Q\left(\tilde{\boldsymbol{\sigma}}_{n+1}\right)}{\partial \tilde{\boldsymbol{\sigma}}_{n+1}} \\
\tilde{\boldsymbol{\sigma}}_{n+1}\left(\Delta \tilde{\lambda}_{n+1}\right) & =\boldsymbol{\sigma}_{n+1}^{\text {trial }}\left(\boldsymbol{\varepsilon}_{n+1}\right)-\Delta \tilde{\lambda}_{n+1} \mathbf{C} \frac{\partial Q\left(\tilde{\boldsymbol{\sigma}}_{n+1}\right)}{\partial \tilde{\boldsymbol{\sigma}}_{n+1}},
\end{aligned}
$$

where $\Delta \tilde{\lambda}_{n+1}=\Delta \lambda_{n}$ is the explicit evaluation of the plastic multiplier. Note the use of the symbol ( $)$ above to point out the difference between the implicit internal variables and the IMPL-EX internal variables.

As a result of the update of the internal variables in the IMPL-EX scheme, the tangent constitutive matrix 
$\mathbf{C}^{\tan }\left(\boldsymbol{\sigma}_{n+1}\right)$ defined in Eq. 16 assumes the following form (according to Eq. 17),

$$
\begin{aligned}
\tilde{\mathbf{C}}^{\tan }\left(\tilde{\boldsymbol{\sigma}}_{n+1}\right) & =\frac{\partial \tilde{\boldsymbol{\sigma}}_{n+1}}{\partial \boldsymbol{\varepsilon}_{n+1}} \\
& =\frac{\partial\left(\mathbf{C}\left(\boldsymbol{\varepsilon}_{n+1}-\boldsymbol{\varepsilon}_{n}^{p}\right)-\mathbf{C} \Delta \tilde{\lambda}_{n+1} \frac{\partial Q\left(\tilde{\boldsymbol{\sigma}}_{n+1}\left(\boldsymbol{\varepsilon}_{n+1}\right)\right)}{\partial \tilde{\boldsymbol{\sigma}}_{n+1}}\right)}{\partial \boldsymbol{\varepsilon}_{n+1}} .
\end{aligned}
$$

Note that generally $\tilde{\mathbf{C}}^{\tan }\left(\tilde{\boldsymbol{\sigma}}_{n+1}\right)$ is not constant due to the dependence of the potential function $Q$ on the total strain $\boldsymbol{\varepsilon}_{n+1}\left(Q=Q\left(\tilde{\boldsymbol{\sigma}}_{n+1}\left(\boldsymbol{\varepsilon}_{n+1}\right)\right)\right)$.

Summarizing, the standard IMPL-EX, is a combination of implicit and explicit integration schemes, in which in a representative time step $n+1$, two stresses are computed: one explicitly (the final stresses $\tilde{\boldsymbol{\sigma}}_{n+1}$, expressed in Eq. 17) and one implicitly $\left(\sigma_{n+1}\right.$, expressed by Eq. 15). The explicit stresses $\tilde{\boldsymbol{\sigma}}_{n+1}$ are used to fulfill the balance equation, while the implicit stresses $\sigma_{n+1}$ are used as the updated stresses for $\sigma_{n}$ in the next time step, which suggests that one should still compute $\Delta \boldsymbol{\varepsilon}_{n+1}^{p}$ (according to Eq. $14_{(2)}$ ) but only use it in the next time step $n+1$ for the computation of the trial stresses in Eq. 11.

Note also that the steps presented in subsection 3.1 remain quite the same for the IMPL-EX integration scheme, with slight changes in how the internal variables are updated (step 4) and in how the tangent constitutive matrix is computed (step 5).

\subsection{Modified IMPL-EX integration scheme updates}

In contrast to the standard IMPL-EX integration scheme ([5]), in the Modified IMPL-EX, for the sake of convenience, the internal variables are updated in terms of the explicit evaluation of the components of the increment in the plastic strain tensor $\left(\Delta \tilde{\boldsymbol{\varepsilon}}_{n+1}^{p}=\Delta \boldsymbol{\varepsilon}_{n}^{p}\right)$, instead of the explicit evaluation of the plastic multiplier $\left(\Delta \tilde{\lambda}_{n+1}=\Delta \lambda_{n}\right)$ as explained above.

In other words, as well as the standard IMPL-EX, the Modified IMPL-EX, is a combination of implicit and explicit integration schemes, in which in the same representative time step $n+1$, two stresses are computed: one implicitly $\left(\sigma_{n+1}\right.$, expressed by Eq. 15 , reproduced below)

$\boldsymbol{\sigma}_{n+1}=\boldsymbol{\sigma}_{n+1}^{\text {trial }}-\mathbf{C} \Delta \boldsymbol{\varepsilon}_{n+1}^{p}=\mathbf{C}\left(\boldsymbol{\varepsilon}_{n+1}-\boldsymbol{\varepsilon}_{n}^{p}\right)-\mathbf{C} \Delta \boldsymbol{\varepsilon}_{n+1}^{p}$

and one explicitly (the final stresses $\tilde{\boldsymbol{\sigma}}_{n+1}$ ), conveniently expressed as follows,

$\tilde{\boldsymbol{\sigma}}_{n+1}=\boldsymbol{\sigma}_{n+1}^{\text {trial }}-\mathbf{C} \Delta \tilde{\boldsymbol{\varepsilon}}_{n+1}^{p}=\mathbf{C}\left(\boldsymbol{\varepsilon}_{n+1}-\boldsymbol{\varepsilon}_{n}^{p}\right)-\mathbf{C} \Delta \tilde{\boldsymbol{\varepsilon}}_{n+1}^{p}$,

where $\Delta \tilde{\boldsymbol{\varepsilon}}_{n+1}^{p}=\Delta \boldsymbol{\varepsilon}_{n}^{p}$ is the explicit evaluation of the components of the increment of the plastic strain tensor, while
$\Delta \boldsymbol{\varepsilon}_{n}^{p}$ is the last converged increment of the plastic strain at time step $n+1$.

The advantage of this approach, for elastoplasticity problems, in comparison with the standard IMPL-EX ([5]) approach, is that the tangent constitutive matrix $\mathbf{C}^{\tan }\left(\boldsymbol{\sigma}_{n+1}\right)$, defined in Eq. 16 becomes constant and as a consequence, the global Newton-Raphson algorithm becomes the initial stiffness method.

Comparing the explicit stresses $\tilde{\boldsymbol{\sigma}}_{n+1}$ computed in Eq. 17 and the ones computed in Eq. 19 above one can see that in Eq. 19 the explicit stresses $\tilde{\boldsymbol{\sigma}}_{n+1}$ are computed through the explicit evaluation of the components of the increment of the plastic strain tensor $\left(\Delta \tilde{\boldsymbol{\varepsilon}}_{n+1}^{p}=\Delta \boldsymbol{\varepsilon}_{n}^{p}\right)$, instead of the explicit evaluation of the plastic multiplier $\left(\Delta \tilde{\lambda}_{n+1}=\Delta \lambda_{n}\right)$, as in Eq. 17.

Hence here, we arrived at the key point in the Modified IMPL-EX integration scheme. The Eq. 19, is the expression of the explicit stress update in the Modified IMPLEX, instead of the Eq. 17 in the standard IMPL-EX ([5]). It states that one must explicitly update all of the components of the plastic strain $\Delta \tilde{\boldsymbol{\varepsilon}}_{n+1}^{p}$, while in the IMPL-EX scheme of [5] (Eqs. 17, 18), one must explicitly update only the plastic multiplier $\Delta \tilde{\lambda}_{n+1}$ (a scalar).

Additionally, in the Modified IMPL-EX, as well as in the standard IMPL-EX, the explicit stresses $\tilde{\boldsymbol{\sigma}}_{n+1}$ are used to fulfill the balance equation, while the implicit stresses $\sigma_{n+1}$ are used as the updated stresses for $\sigma_{n}$ in the next time step $n+1$. Therefore, one should also compute $\Delta \boldsymbol{\varepsilon}_{n+1}^{p}$ (according to Eq. $14_{(2)}$ ), but only use it in the next time step $n+1$ for the computation of the trial stresses, according to Eq. 11.

Finally, as has been mentioned before, a consequence of Eq. 19 is that the tangent constitutive matrix $\mathbf{C}^{\tan }\left(\sigma_{n+1}\right)$, defined in Eq. 16, becomes constant, i.e., independent of the current strain $\boldsymbol{\varepsilon}_{n+1}$ (differently from Eq. 18),

$\tilde{\mathbf{C}}^{\tan }=\frac{\partial \tilde{\boldsymbol{\sigma}}_{n+1}}{\partial \boldsymbol{\varepsilon}_{n+1}}=\frac{\partial\left(\mathbf{C}\left(\boldsymbol{\varepsilon}_{n+1}-\boldsymbol{\varepsilon}_{n}^{p}\right)-\mathbf{C} \Delta \tilde{\boldsymbol{\varepsilon}}_{n+1}^{p}\right)}{\partial \boldsymbol{\varepsilon}_{n+1}}=\mathbf{C}$

making the tangent stiffness matrix $\mathbf{K}_{n+1}$ in Eq. 3 equal to the constant elastic tangent stiffness matrix throughout the analysis. Note that the features of the constant elastic tangent stiffness matrix, i.e., its symmetry and positive definiteness, guarantee the unconditional stability of the modified IMPL-EX integration scheme.

\subsection{Comparison between the standard and modified IMPL-EX}

In Table 1 below, a comparison between the steps necessary for the integration of the nonlinear evolution of the stresses at the integration point level of the Modified IMPL-EX scheme and the standard IMPL-EX scheme is made. 
Table 1 Standard IMPL-EX and the modified IMPL-EX steps at the integration point level

\begin{tabular}{lll}
\hline Steps & Standard IMPL-EX & Modified IMPL-EX \\
\hline 1-trial stress computation & $\boldsymbol{\sigma}_{n+1}^{\text {trial }}=\boldsymbol{\sigma}_{n}+\mathbf{C} \Delta \boldsymbol{\varepsilon}_{n+1}$ & $\boldsymbol{\sigma}_{n+1}^{\text {trial }}=\boldsymbol{\sigma}_{n}+\mathbf{C} \Delta \boldsymbol{\varepsilon}_{n+1}$ \\
2-yield function evaluation & $F=F^{\text {trial }}\left(\boldsymbol{\sigma}_{n+1}^{\text {trial }}, \boldsymbol{\alpha}_{n}\right)$ & $F=F^{\text {trial }}\left(\boldsymbol{\sigma}_{n+1}^{\text {trial }}, \boldsymbol{\alpha}_{n}\right)$ \\
3-implicit plastic strain increment & $\Delta \boldsymbol{\varepsilon}_{n+1}^{p}=\Delta \lambda_{n+1} \frac{\partial Q\left(\boldsymbol{\sigma}_{n+1}\right)}{\partial \boldsymbol{\sigma}_{n+1}}$ & $\Delta \boldsymbol{\varepsilon}_{n+1}^{p}=\Delta \lambda_{n+1} \frac{\partial Q\left(\boldsymbol{\sigma}_{n+1}\right)}{\partial \boldsymbol{\sigma}_{n+1}}$ \\
4-explicit plastic strain evaluation & $\Delta \tilde{\lambda}_{n+1}=\Delta \lambda_{n}$ & $\Delta \tilde{\boldsymbol{\varepsilon}}_{n+1}^{p}=\Delta \boldsymbol{\varepsilon}_{n}^{p}$ \\
& $\tilde{\boldsymbol{\alpha}}_{n+1}\left(\Delta \tilde{\lambda}_{n+1}\right)=\boldsymbol{\alpha}_{n}+\boldsymbol{\varphi}_{n}\left(\Delta \tilde{\lambda}_{n+1}\right)$ & $\tilde{\boldsymbol{\varepsilon}}_{n+1}^{p}=\boldsymbol{\varepsilon}_{n}^{p}+\Delta \tilde{\boldsymbol{\varepsilon}}_{n+1}^{p}$ \\
& $\tilde{\boldsymbol{\varepsilon}}_{n+1}^{p}=\boldsymbol{\varepsilon}_{n}^{p}+\Delta \tilde{\lambda}_{n+1} \mathbf{C} \frac{\partial Q\left(\tilde{\boldsymbol{\sigma}}_{n+1}\right)}{\partial \tilde{\boldsymbol{\sigma}}_{n+1}}$ & $\boldsymbol{\sigma}_{n+1}=\boldsymbol{\sigma}_{n+1}^{\text {trial }}-\mathbf{C} \Delta \boldsymbol{\varepsilon}_{n+1}^{p}$ \\
5-implicit stress evaluation & $\boldsymbol{\sigma}_{n+1}=\boldsymbol{\sigma}_{n+1}^{\text {trial }}-\mathbf{C} \Delta \boldsymbol{\varepsilon}_{n+1}^{p}$ & $\tilde{\boldsymbol{\sigma}}_{n+1}=\boldsymbol{\sigma}_{n+1}^{\text {trial }}-\mathbf{C} \Delta \tilde{\boldsymbol{\varepsilon}}_{n+1}^{p}$ \\
6-explicit stress evaluation & $\tilde{\boldsymbol{\sigma}}_{n+1}\left(\Delta \tilde{\lambda}_{n+1}\right)=\boldsymbol{\sigma}_{n+1}^{\text {trial }}\left(\boldsymbol{\varepsilon}_{n+1}\right)-\Delta \tilde{\lambda}_{n+1} \mathbf{C} \frac{\partial Q\left(\tilde{\boldsymbol{\sigma}}_{n+1}\right)}{\partial \tilde{\boldsymbol{\sigma}}_{n+1}}$ & $\tilde{\mathbf{C}}^{\text {tan }}=\frac{\partial\left(\mathbf{C}\left(\boldsymbol{\varepsilon}_{n+1}-\boldsymbol{\varepsilon}_{n}^{p}\right)-\mathbf{C} \Delta \tilde{\boldsymbol{\varepsilon}}_{n+1}^{p}\right)}{\partial \boldsymbol{\varepsilon}_{n+1}}=\mathbf{C}$ \\
7-tangent constitutive matrix & $\tilde{\mathbf{C}}^{\text {tan }}\left(\tilde{\boldsymbol{\sigma}}_{n+1}\right)=\frac{\partial\left(\mathbf{C}\left(\boldsymbol{\varepsilon}_{n+1}-\boldsymbol{\varepsilon}_{n}^{p}\right)-\mathbf{C} \Delta \tilde{\lambda}_{n+1} \frac{\partial Q\left(\tilde{\boldsymbol{\sigma}}_{n+1}\left(\boldsymbol{\varepsilon}_{n+1}\right)\right)}{\partial \tilde{\boldsymbol{\sigma}}_{n+1}}\right)}{\partial \boldsymbol{\varepsilon}_{n+1}}$ & ${ }^{2}$ \\
\hline
\end{tabular}

\section{Examples}

To assess the accuracy, robustness and computability of the modified IMPL-EX, four benchmark numerical examples with analytical solutions are presented in what follows.

The yield function $F=F(\sigma, \alpha)$ for each of the yield criteria used in the following analyses are presented in Table 2 below,

Where $K$ is the plastic modulus, $\alpha$ is the internal hardening variable, $I_{1}$ is the first invariant of the stress tensor, $J_{2}$ is the second invariant of the deviatoric stress tensor, $\theta$ $\left(-\frac{\pi}{6} \leq \theta \leq \frac{\pi}{6}\right)$ is the Lode angle, $\phi$ is the frictional angle, $c$ is the cohesion and $\sigma_{Y}$ is the uniaxial yield stress.

The corner problem in Tresca and Mohr-Coulomb yield criteria is dealt with using the equations presented in Table 3 shown below,

Table 2 Yield criteria used in the analyses

\begin{tabular}{ll}
\hline Criterion & Expressions for $F=F(\sigma, \alpha)$ \\
\hline von Mises & $F=\sqrt{3 J_{2}}-\left(\sigma_{Y}+K \alpha\right)$ \\
Tresca & $F=2 \sqrt{J_{2}} \cos (\theta)-\left(\sigma_{Y}+K \alpha\right)$ \\
Drucker-Prager & $F=\eta \frac{I_{1}}{3}+\sqrt{J_{2}}-\xi c$ \\
& $\eta=\frac{6 \sin \phi}{\sqrt{3}(3+\sin \phi)} \quad \xi=\frac{6 \cos \phi}{\sqrt{3}(3+\sin \phi)}$ \\
Mohr-Coulomb & $\left.\quad-\frac{1}{\sqrt{3}} \sin \theta \sin \phi\right)-c \cos (\phi)$ \\
&
\end{tabular}

Table 3 Yield criteria functions at the corner

\begin{tabular}{lr}
\hline Criterion & Expressions for $F=F(\sigma, \alpha)$ \\
\hline Tresca & $F=\sqrt{3 J_{2}}-\left(\sigma_{Y}+K \alpha\right)$ \\
Mohr-Coulomb & $F=\frac{I_{1} \sin (\phi)}{3}+\frac{\sqrt{3 J_{2}}}{2}(1+$ \\
& $\left.\pm \frac{\sin (\phi)}{3}-c \cos (\phi)\right)$ \\
\hline
\end{tabular}

\subsection{Uniformly loaded circular plate}

In this axisymmetric example, a simply supported uniformly loaded circular plate is analyzed using an elastic perfectly plastic material model with a von Mises yield criterion. The purpose of the analysis was to determine the limit load $P_{\text {lim }}$ for the plate and compare the results obtained using the modified IMPL-EX with the results obtained using implicit schemes (full Newton-Raphson and initial stiffness). The analytical value of the limit load (see, e.g., [2]), for the material parameters given in Table 4 and for the geometry, the boundary conditions and the load configuration given in Fig. 1 is $P_{\text {lim }}=260.8$.

The Fig. 1 shows the finite element mesh with ten eightnoded axisymmetric quadrilaterals elements. For the stress computation, a $2 \times 2$ Gauss integration point scheme was adopted.

Table 4 Material properties

\begin{tabular}{ll}
\hline von Mises model & \\
\hline Young's modulus $E$ & $10^{7}$ \\
Poisson's ratio $v$ & 0.24 \\
Yield stress $\sigma_{Y}$ & 16,000 \\
Plastic modulus $K$ & 0 \\
\hline
\end{tabular}

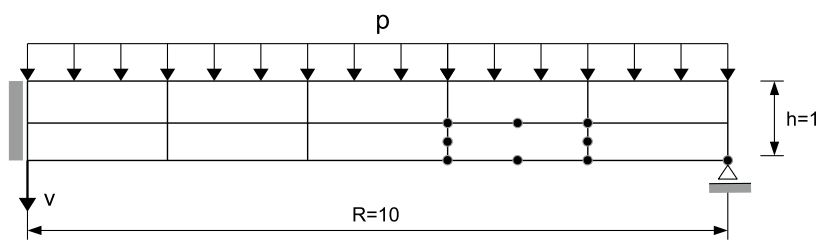

Fig. 1 Uniformly loaded circular plate. Geometry, boundary conditions and load 


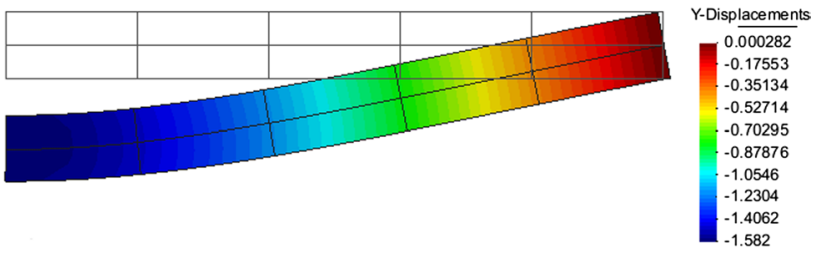

Fig. 2 Uniformly loaded circular plate. Deformed structure at step 80 (IMPL-EX analysis)

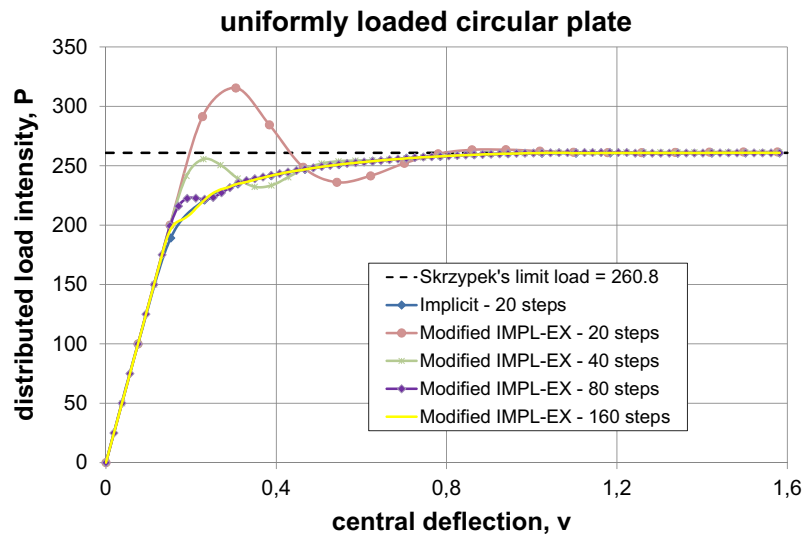

Fig. 3 Uniformly loaded circular plate. Load versus deflection diagram

Figure 2 shows the deformed configuration of the uniformly loaded circular plate obtained with the modified IMPL-EX scheme. The value obtained for the maximum $Y$-displacement $\left(d_{y}=-1.582\right)$ is the same for the modified IMPL-EX scheme using 20 load steps and the implicit scheme. Also, the limit load obtained with the modified IMPL-EX scheme and the implicit scheme is the same. This show that despite the slight difference in the displacement-load curve obtained with the modified IMPL-EX, the final results are very accurate (see Fig. 3). The results obtained with various numbers of load steps for the modified IMPL-EX scheme and the implicit scheme are presented in the graphics and tables that follow.
Figure 3 shows a graphic of the deflection, v, at the center of the plate plotted against the applied pressure $P$. Note the accuracy of the modified IMPL-EX in capturing the collapse of the plate, even with the use of a small number of load steps. Note also the oscillations of the modified IMPL-EX results for intermediate loads (especially for abrupt changes in the elastic/plastic stress state) when a small number of load steps (large time steps) are used. This drawback can be overcome by using a greater number of load steps (small time steps), as shown in Fig. 3.

Table 5 compares the performance of the modified IMPL-EX integration scheme against fully implicit methods (the full Newton-Raphson method and the Initial Stiffness method). Note the tremendous reduction in the total number of iterations and computation time with respect to the Initial Stiffness method when the modified IMPL-EX integration scheme is used.

Note also that even with 8 times more load steps (160 load steps), the modified IMPL-EX integration scheme still represents a huge reduction in the total number of iterations (and hence in calculation time) (see Table 5); Note that, with 160 load steps the displacement-load curve obtained with the Modified IMPL-EX has a very good agreement (accuracy) with the reference results (see Fig. 3).

\subsection{Plate with hole}

In this example, a plane strain analysis of a rectangular plate with a center hole loaded vertically beyond the elastic range is carried out. For symmetry reasons, only one-fourth of the plate is analyzed, as shown in Fig. 4. The material model used in this analysis is an elastoplastic von Mises yield criterion with linear strain hardening/softening. The material parameters of the analysis are shown in Table 6 .

Figure 5 below shows a comparison between the errors of analysis provided by the implicit integration scheme, the modified IMPL-EX scheme and the IMPL-EX ([5]) scheme. The error was obtained in terms of the $L_{2}$ norms of the corresponding force-displacement curve, $F-v$, as follows,

error $=\frac{\left\|F_{\mathrm{NSTEP}}-F_{\infty}\right\|}{\left\|F_{\infty}\right\|}$
Table 5 Total number of iterations of the uniformly loaded circular plate analysis

\begin{tabular}{|c|c|c|c|c|c|c|}
\hline \multirow[t]{3}{*}{ No. of load steps } & \multicolumn{6}{|c|}{ Total number of iterations } \\
\hline & \multicolumn{2}{|c|}{$\begin{array}{l}\text { Full Newton-Raphson } \\
\text { method }\end{array}$} & \multicolumn{2}{|c|}{ Initial stiffness method } & \multicolumn{2}{|c|}{$\begin{array}{l}\text { Modified IMPL-EX } \\
\text { scheme }\end{array}$} \\
\hline & Iter. & Time (s) & Iter. & Time (s) & Iter. & Time (s) \\
\hline 20 & 66 & 0.07 & 4904 & 1.68 & 38 & 0.04 \\
\hline 40 & 114 & 0.12 & 8271 & 3.07 & 76 & 0.08 \\
\hline 80 & 203 & 0.22 & 14,809 & 5.15 & 153 & 0.13 \\
\hline 160 & 361 & 0.39 & 26,889 & 9.00 & 306 & 0.29 \\
\hline
\end{tabular}



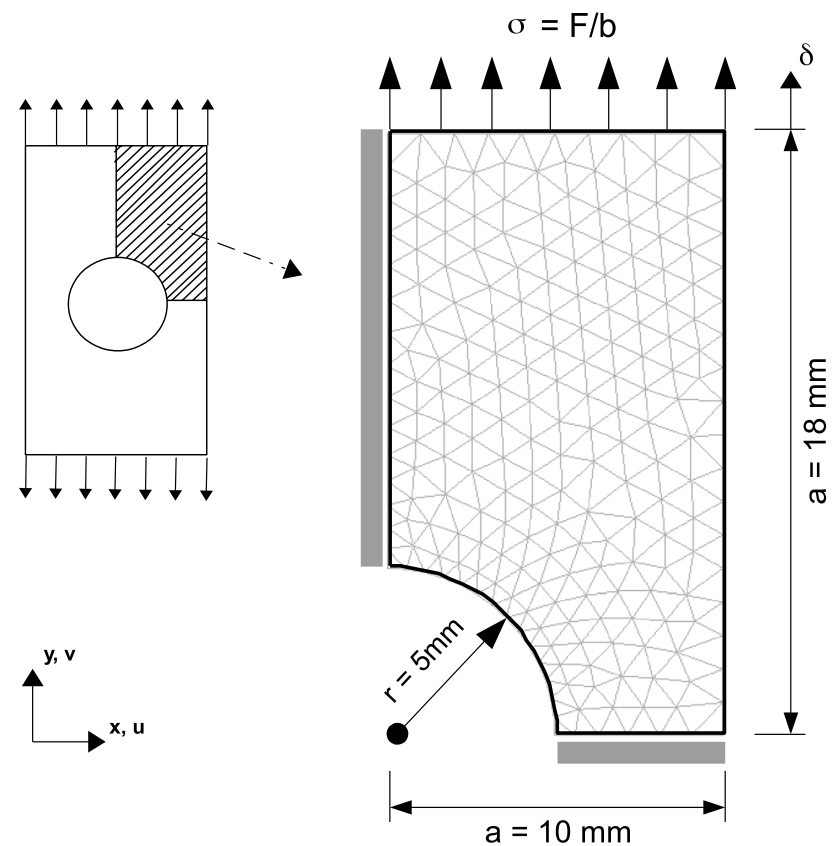

Fig. 4 Plate with hole. Geometry, boundary conditions and load

Table 6 Material properties

\begin{tabular}{ll}
\hline von Mises model & \\
\hline Young's modulus $E$ & $70 \mathrm{MPa}$ \\
Poisson's ratio $v$ & 0.2 \\
Yield stress $\sigma_{Y}$ & $0.24 \mathrm{MPa}$ \\
Plastic modulus $K$ & $2.24 \mathrm{MPa}$ (hardening) \\
& $-1.0 \mathrm{MPa}$ (softening) \\
\hline
\end{tabular}

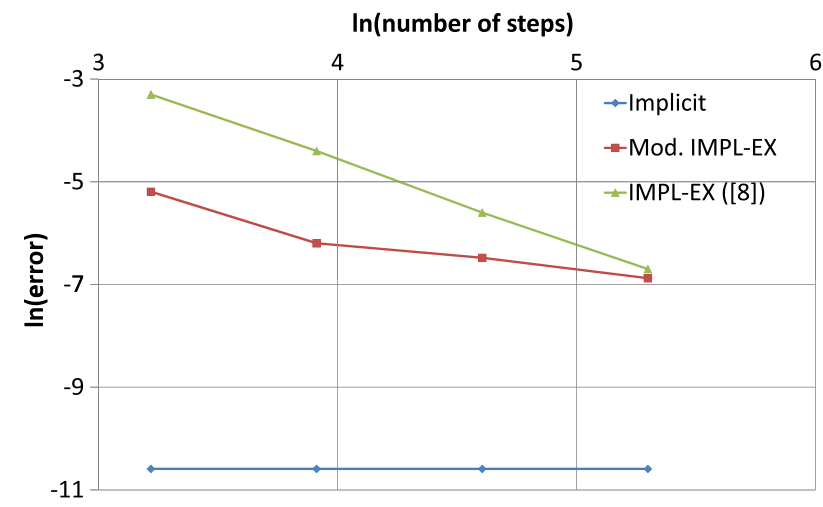

Fig. 5 Plate with hole. Error (log-log) diagram

where NSTEP is the number of steps used for the complete analysis and $F_{\infty}$ is referenced to the exact solution obtained with a large value of NSTEP. The error was obtained for the stage of the analysis in which the displacement-load

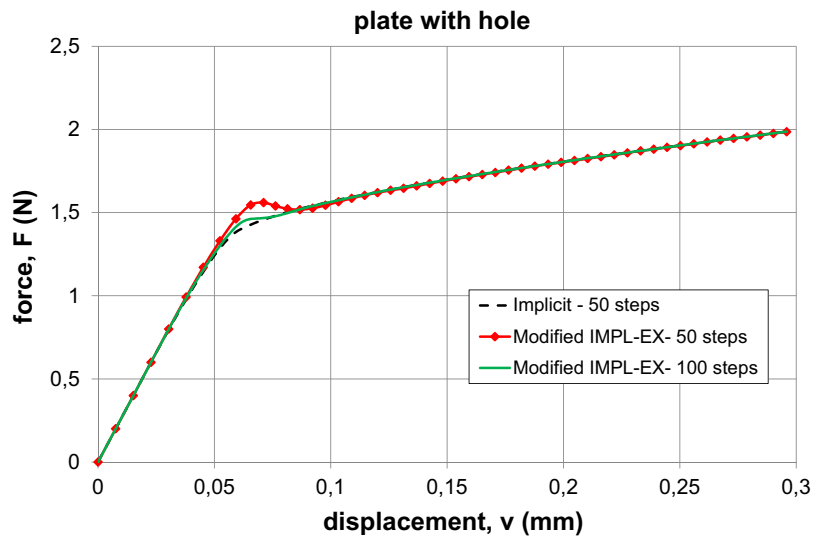

Fig. 6 Plate with hole. Force-displacement diagram with hardening scenario

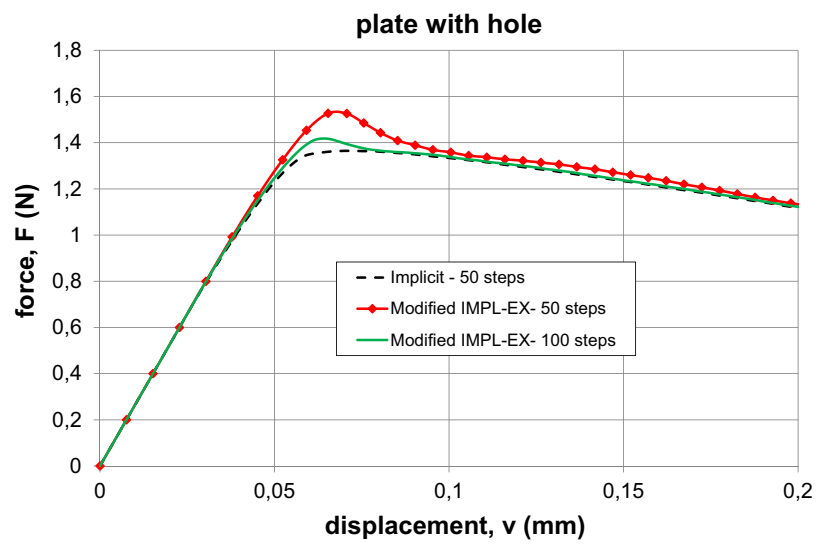

Fig. 7 Plate with hole. Force-displacement diagram with softening scenario

curve drifts from the true solution, i.e., the stage where the stresses pass from elastic to plastic state.

The force-displacement curves, $F-v$, obtained with both the implicit and modified IMPL-EX integration schemes are presented in Fig. 6 for hardening analysis and in Fig. 7 for softening analysis to show that the algorithm can be used for both.

Table 7 shows a comparison of the total number of iterations and computation time (in seconds) between the implicit integration schemes (full Newton-Raphson and initial stiffness method) and the modified IMPL-EX integration scheme for hardening analysis. Note that, despite the exceptional performance of the full Newton-Raphson method in this example (due to the bilinear behavior of the force-displacement diagram shown in Fig. 6), the modified IMPL-EX scheme shows impressive performance (in number of iterations and time) and even better performance when compared with the implicit initial stiffness method. 
Table 7 Total number of iterations of the plate with hole analysis

\begin{tabular}{|c|c|c|c|c|c|c|}
\hline \multirow[t]{3}{*}{ No. of load steps } & \multicolumn{6}{|c|}{ Total number of iterations } \\
\hline & \multicolumn{2}{|c|}{$\begin{array}{l}\text { Full Newton-Raphson } \\
\text { method }\end{array}$} & \multicolumn{2}{|c|}{ Initial stiffness scheme } & \multicolumn{2}{|c|}{$\begin{array}{l}\text { Modified IMPL-EX } \\
\text { scheme }\end{array}$} \\
\hline & Iter. & Time (s) & Iter. & Time (s) & Iter. & Time (s) \\
\hline 50 & 74 & 2.22 & 711 & 5.33 & 96 & 1.40 \\
\hline 100 & 115 & 2.70 & 950 & 8.11 & 193 & 2.89 \\
\hline
\end{tabular}

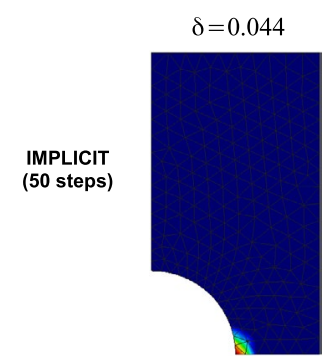

$\delta=0.058$
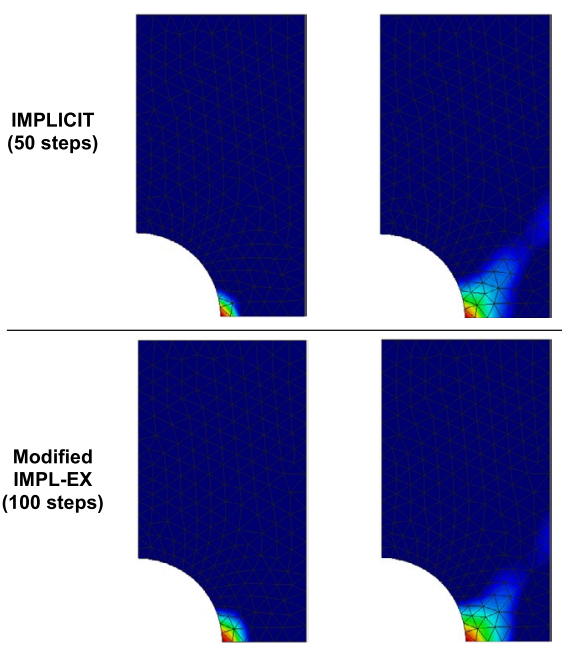

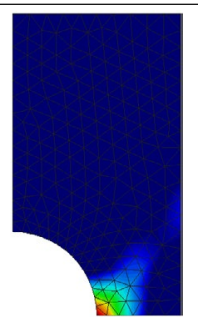

$\delta=0.154$
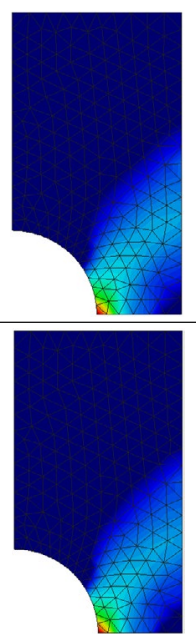
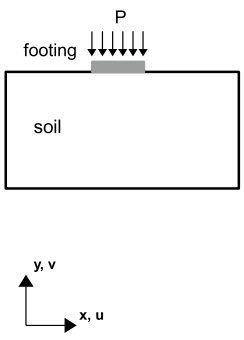

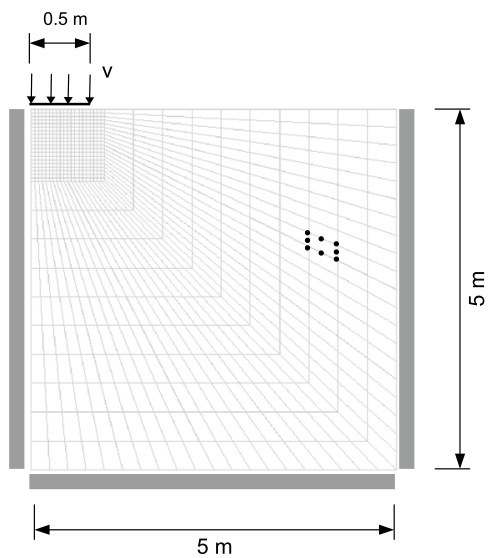

Fig. 9 Strip-footing collapse. Geometry, boundary conditions and load
Fig. 8 Plate with hole. Evolution of the plastic region with von Mises criterion. Implicit scheme with 50 load steps and IMPL-EX scheme with 100 load steps

The evolution of the plastic region, also obtained with the implicit and modified IMPL-EX schemes, for hardening analysis is shown in Fig. 8.

\subsection{Strip-footing collapse}

With respect to this example, [2] determines the bearing capacity (limit load) of a strip footing with a width $B=1 \mathrm{~m}$ and length $L=5 \mathrm{~m}$ lying on soil (assumed as an infinite medium), according to Fig. 9.

The soil is assumed to be weightless, and the analysis is carried out with three elasto-perfect plastic material models (Tresca, Mohr-Coulomb and Drucker-Prager). The material parameters and the collapse pressure $P_{\text {lim }}$ (see [2]) for each material model are shown in Table 8 . Note that, for the Tresca model analysis, $\sigma_{Y}=2 c$.

In Fig. 10 the strip footing obtained with the modified IMPL-EX using 100 load steps and the Mohr-Coulomb model is shown.

In Figs. 11, 12 and 13 diagrams of the normalized average pressure $P / c$ versus the normalized settlement $u / B$ obtained by the analyses using the Tresca, Mohr-Coulomb
Table 8 Material properties and collapse pressure

\begin{tabular}{ll}
\hline Tresca model & \\
Young's modulus $E$ & $10^{7} \mathrm{kPa}$ \\
Poisson's ratio $v$ & 0,48 \\
Yield stress $\sigma_{Y}$ & $980 \mathrm{kPa}$ \\
Plastic modulus $K$ & 0 \\
$P_{\text {lim }} / c$ & 5.14 \\
Mohr-Coulomb model & \\
Young's modulus $E$ & $10^{7} \mathrm{kPa}$ \\
Poisson's ratio $v$ & 0,48 \\
Cohesion $c$ & $490 \mathrm{kPa}$ \\
$P_{\text {lim }} / c$ & 14.8 \\
Drucker-Prager model & \\
Young's modulus $E$ & $10^{7} \mathrm{kPa}$ \\
Poisson's ratio $v$ & 0,48 \\
Cohesion $c$ & $490 \mathrm{kPa}$ \\
$P_{\text {lim }} / c$ & 14.8 \\
\hline
\end{tabular}

and Drucker-Prager models, respectively, are plotted. Note the good agreement between the results obtained with the implicit and with the modified IMPL-EX schemes with at most 50, 100 and 200 steps. 


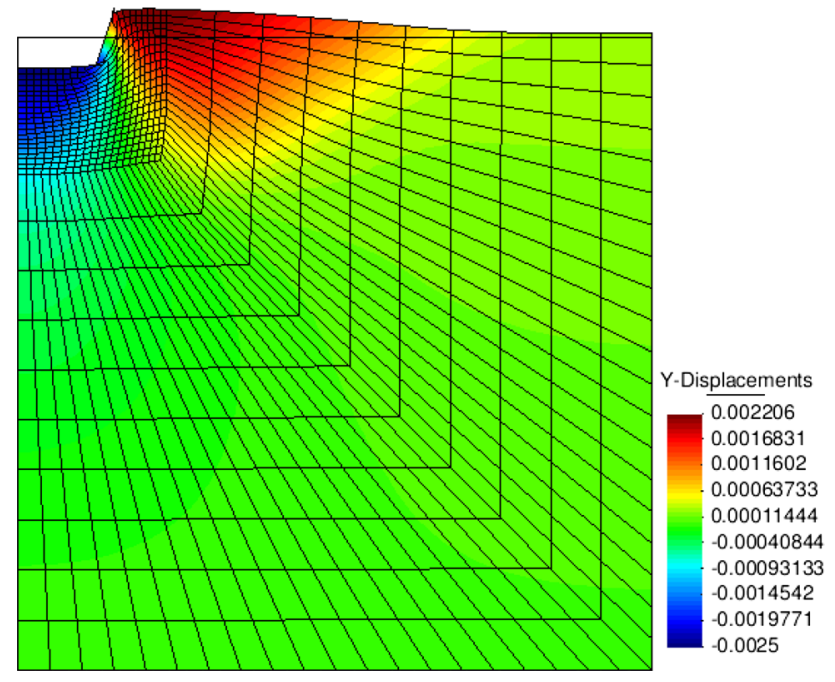

Fig. 10 Strip-footing collapse. Incremental nodal displacement field at collapse for modified IMPL-EX scheme analysis with 100 steps

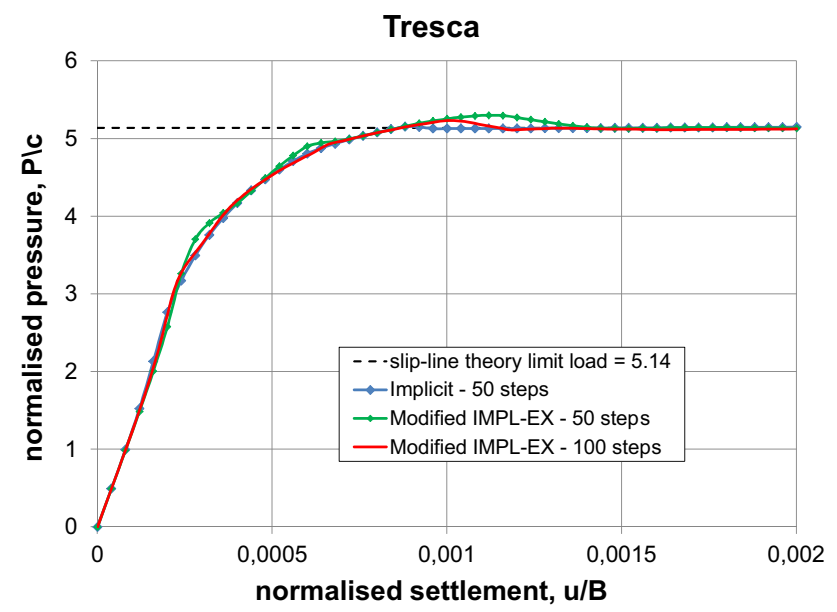

Fig. 11 Strip-footing collapse. Load versus displacement diagram for Tresca model

\subsection{Internally pressurized cylinder}

In this plane strain example, [2] simulates a long metallic thick-walled cylinder subjected to internal pressure, as shown in Fig. 14. For symmetry reasons, only one-fourth of the cylinder is analyzed. For the finite element mesh, standard eight-noded quadrilateral elements with four $(2 \times 2)$ Gauss integration points are adopted.

For the material model, an elastic perfectly plastic von Mises yield criterion was adopted. The material properties and yield criterion parameters used in the analysis are shown in the Table 9.

The collapse pressure $P_{\lim }$ according to [3] is $P_{\text {lim }} \approx 0.19209 \mathrm{GPa}$. Figure 15 demonstrates the very good

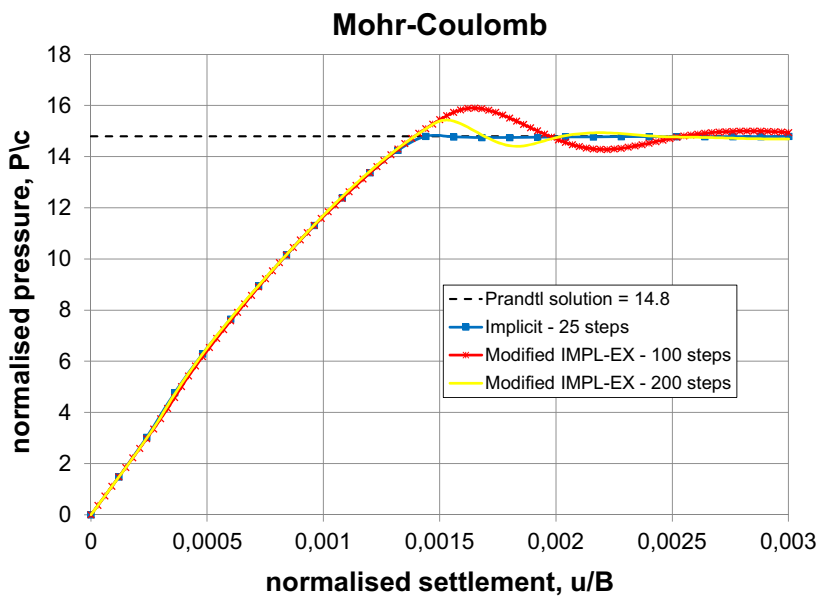

Fig. 12 Strip-footing collapse. Load versus displacement diagram for Mohr-Coulomb model

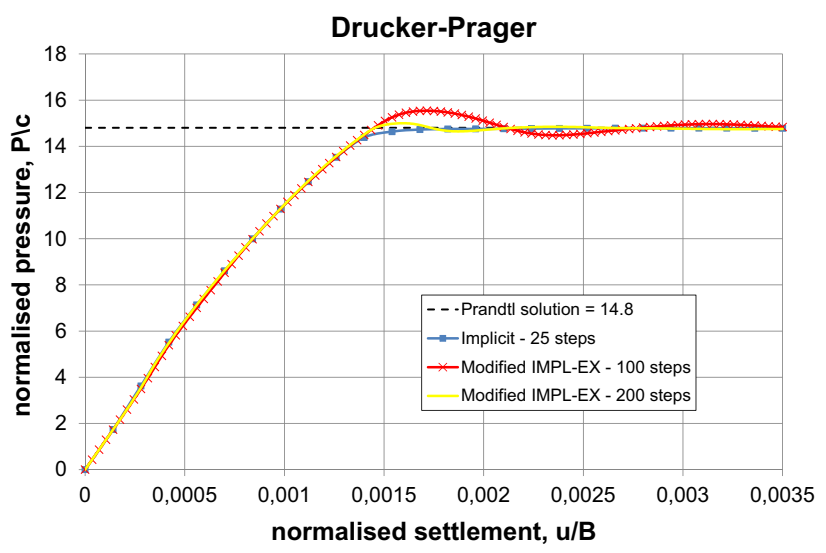

Fig. 13 Strip-footing collapse. Load versus displacement diagram for Drucker-Prager model

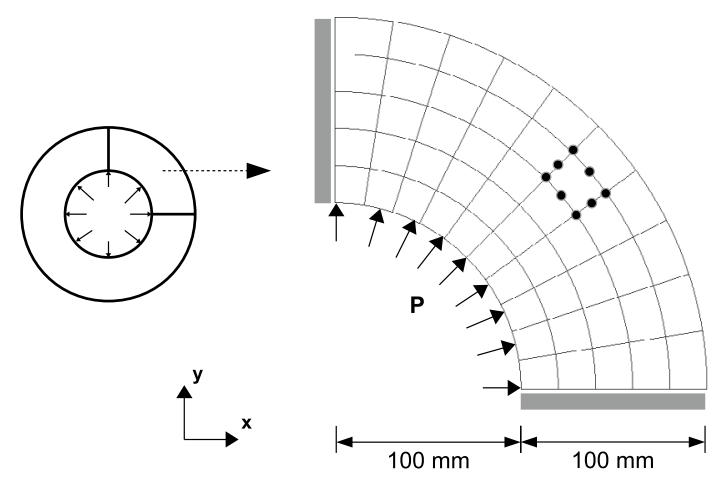

Fig. 14 Internally Pressurized Cylinder. Geometry and load

agreement between the collapse pressure obtained with the modified IMPL-EX (using 25 and 50 load steps only) and the one obtained with the closed-form solution of [3]. 
Table 9 Material properties

\begin{tabular}{ll}
\hline Von Mises model & \\
Young's modulus $E$ & $210 \mathrm{GPa}$ \\
Poisson's ratio $v$ & 0.3 \\
Yield stress $\sigma_{Y}$ & $0.24 \mathrm{GPa}$ \\
Plastic modulus $K$ & 0 \\
\hline
\end{tabular}

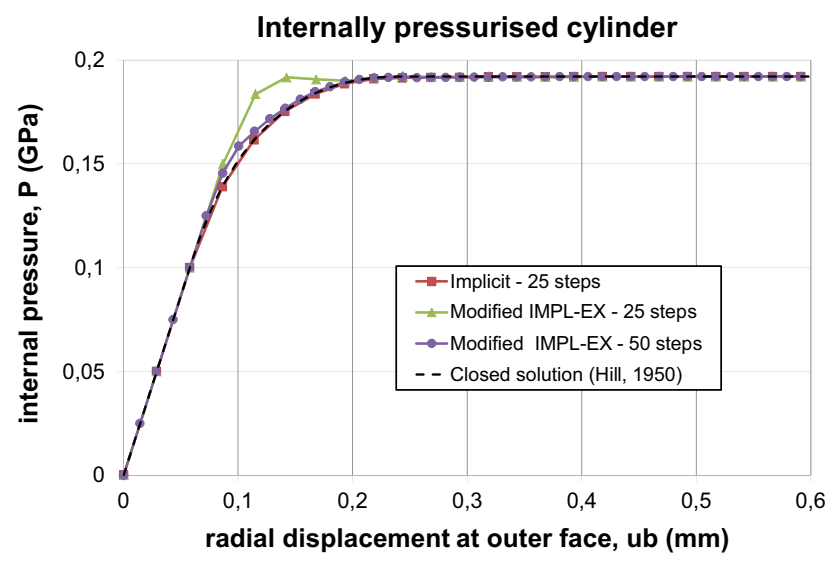

Fig. 15 Internally pressurized cylinder. Pressure versus displacement diagram

In Figs. 16 and 17 below, the hoop and radial stress distributions at different levels of applied internal pressure are shown, demonstrating the accuracy of the modified IMPLEX scheme for the computation of stress.

\section{Conclusion}

In this paper, a novel modified version of the implicitexplicit integration scheme-IMPL-EX-named as Modified IMPL-EX is presented. It is shown that the main difference between the proposed integration scheme and the standard IMPL-EX is the choice of internal variables to be updated in the time step $n+1$. In the Modified IMPLEX it was chosen to update each one of the components of the plastic strain tensor instead of updating the plastic multiplier.

With that update choice the tangent constitutive matrix $\mathbf{C}^{\tan }\left(\sigma_{n+1}\right)$, defined in Eq. 16, becomes constant, i.e., independent of the current strain $\boldsymbol{\varepsilon}_{n+1}$, making the tangent stiffness matrix $\mathbf{K}_{n+1}$ in Eq. 3 equal to the constant elastic tangent stiffness matrix throughout the analysis, and so given it the property of positive definiteness.

To assess the robustness, accuracy and computational cost of the algorithm, four benchmark numerical examples with closed-form solutions are presented. The examples are

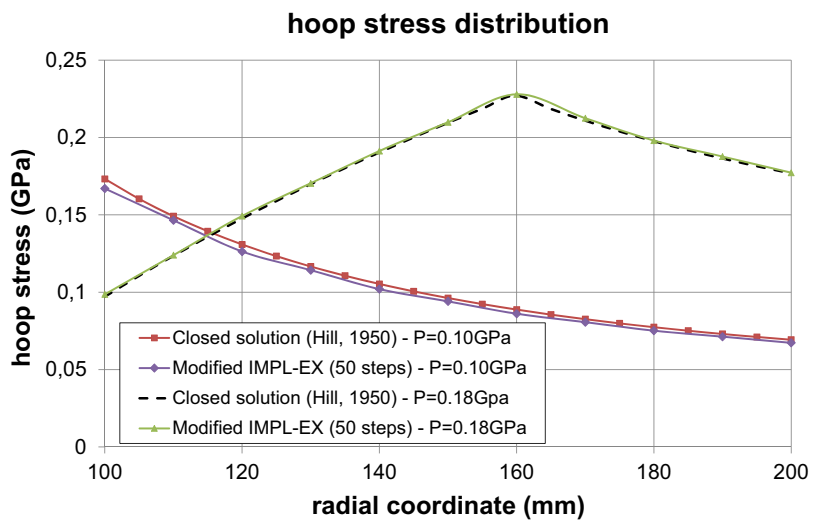

Fig. 16 Internally pressurized cylinder. Hoop stress distributions at different levels of applied internal pressure. Finite element results are computed at Gauss integration points

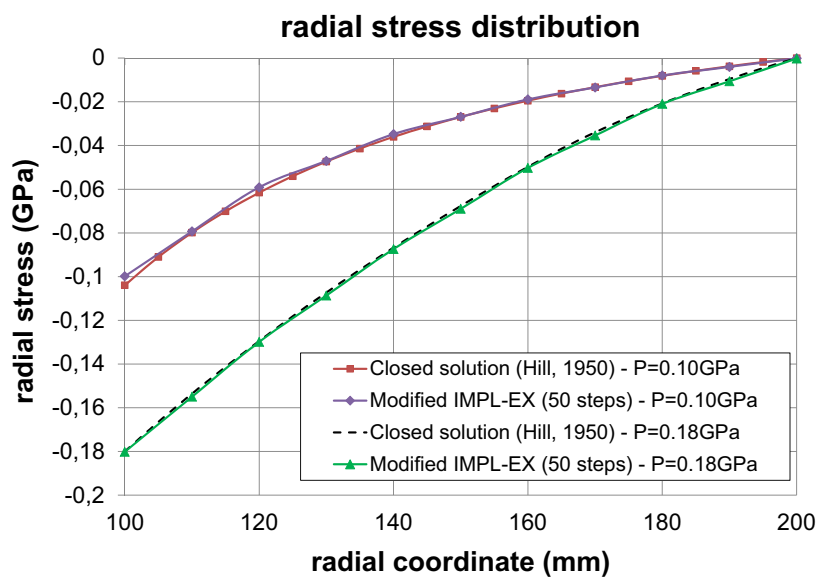

Fig. 17 Internally pressurized cylinder. Radial stress distributions at different levels of applied internal pressure. Finite element results are computed at Gauss integration points

conducted using the most common plasticity models (von Mises, Tresca, Drucker-Prager and Mohr-Coulomb yield criteria).

The examples show that the modified IMPL-EX integration reduces computational costs (Tables 5 and 7). They also show that with the modified IMPL-EX, one can obtain very accurate results with much fewer iterations than one would obtain with the initial stiffness method with the same number of load steps since, with the modified IMPL-EX only two iterations (at most) are performed in each load step. The similarity in accuracy using the IMPL-EX scheme is confirmed by the error estimation presented in the error $(\log -\log )$ diagram shown in example 5.2. The accuracy of the algorithm can always be increased by increasing the number of time steps used 
in the analysis, which also reduces the oscillations in the intermediate load-deformation response, as shown in the examples.

The robustness of the modified IMPL-EX algorithm is guaranteed by the use of the constant elastic tangent stiffness matrix (which is symmetric and positive definite) throughout the analysis, as a result of the explicit integration of the stresses, limiting the number of iteration to at most two in each load step.

Finally, although the algorithm presented in this paper was only applied to elastoplasticity problems, the algorithm is not limited to such problems; in fact, the authors are currently working on another similar paper, to be submitted soon, in which not only elastoplasticity but also the problem of damage mechanics is tackled using the modified IMPL-EX in a manner very similar to that presented here.

Acknowledgments The work presented is a result of a research initiative between the University of São Paulo (USP) and the São Paulo State University (UNESP). The authors of this work are supported by the São Paulo Research Foundation (FAPESP), to which they gratefully acknowledged.

\section{References}

1. Chen C (1992) Efficient and reliable accelerated constant siffness algorithms for the solution of non-linear problems. Int $\mathbf{J}$ Numer Methods Eng 35:481-490

2. de Souza Neto EA, Peric D, Owen DRJ (2008) Computational methods for plasticity - Theory and Applications. Wiley, New York

3. Hill R (1950) The Mathematical Theory of Plasticity. Oxford Unit Press, Oxford

4. Oliver J, Huespe AE, Blanco S, Linero DL (2006) Stability and robustness issues in numerical modeling of material failure with the strong discontinuity approach. Comput Methods Appl Mech Eng 195:7093-7114

5. Oliver J, Huespe AE, Cante JC (2008) An implicit/explicit integration scheme to increase computability of non-linear material and contact/friction problems. Comput Methods Appl Mech Eng 197:1865-1889

6. NS Ottosen, Ristinmaa M (2005) The mechanics of constitutive modeling. Elsevier, Amsterdam

7. Simo JC, Hugles TJR (1998) Computational Inelasticity. Springer, New York

8. Sloan Scott W (1987) Substepping schemes for the numerical integration of elastoplastic stress-strain relations. Int $\mathrm{J}$ Numer Num Meth Eng 24:893-911

9. Sloan Scott W, Abbo Andrew J, Sheng Daichao (2001) Refined explicit integration of elastoplastic models with automatic error control. Eng Comput 18(1/2):121-154 